\title{
Contribution à l'étude des entérocoques et de leurs aptitudes technologiques ${ }^{(1)}$
}

\author{
par \\ J. L. SCHMIDT et J. LENOIR \\ Laboratoire de Recherches de la Chaire de Technologie (I.N.R.A.) \\ Institut National Agronomique Paris-Crignon - Centre de Grignon (78)
}

\section{INTRODUCTION}

Diminuer ses prix de revient, améliorer et régulariser la qualité de sa production sont deux objectifs que l'industrie fromagère ne peut dissocier. Dans cette perspective, plusieurs voies d'expérimentation sont actuellement ouvertes, notamment le développement de la mécanisation et de l'automatisation, la recherche et la mise au point de levains mieux adaptés aux besoins des fabrications fromagères.

La préparation des caillés tend en effet à s'effectuer, de plus en plus, selon des procédés mécanisés. Ceux-ci présentent des avantages techniques et économiques évidents, diminution sensible des besoins en main-d'œuvre, plus grande régularité dans la qualité des fabrications, réduction des temps technologiques... Mais, pour certains types de fromages, notamment les pâtes molles, la mécanisation se heurte à des difficultés inhérentes à la nature même de ces pâtes. Faut-il rappeler que les caillés de pâtes molles doivent leurs caractères typiques au fait qu'ils ne subissent, au cours de leur préparation, qu'un travail mécanique très limité ? L'égouttage est donc lent et l'acidification poussée (Veisseyre, 1966). Une accélération mécanique de l'égouttage tend à se traduire par une insuffisance de l'action microbienne et les fabrications prennent alors un caractère présure trop marqué. Pour conserver aux caillés leurs caractères typiques essentiels il convient donc de respecter une certaine concordance entre l'action des bactéries lactiques au sein de la pâte et le déroulement de l'égouttage. En d'autres termes, l'accélération de l'égouttage doit s'accompagner d'une accélération de la fermentation. A ce niveau se pose donc le problème de l'activité acidifiante du levain.

(1) Travail réalisé dans le cadre d'un contrat de la Délégation Générale à la Recherche Scientifique et Technique. Contrat $\mathrm{n}^{\circ} 6901935$ sur la mise au point d'une Technologie mécanisée de fabrication des pâtes molles. 
Les bactéries du levain, en fromagerie, n'ont pas pour seul rôle l'acidification de la pâte. Par production d'enzymes, elles participent à l'affinage du fromage et au développement de la saveur et de l'arôme (Kosikowski et Mocquot, 1958 ; Jacquet et Lenoir, 1969). Or, la seule intervention des levains lactiques n'est généralement pas suffisante pour assurer au fromage des qualités organoleptiques entièrement satisfaisantes. Il convient donc de développer et de généraliser l'emploi de levains spécialement conçus pour la fromagerie, de levains caractérisés par la présence, à côté des bactéries lactiques, d'une flore complémentaire contrôlée et adaptée au type de fabrication. Ainsi devrait pouvoir être conférée aux fromages de lait pasteurisé une richesse de saveur et d'arôme comparable à celle des bons fromages de lait cru.

A ce double point de vue, accélération du processus d'acidification et développement de qualités organoleptiques plus satisfaisantes, l'emploi dans les levains de fromagerie d'entérocoques pourrait présenter un certain intérêt.

Dans la classification de Bergey (Breed et al., 1957), les entérocoques appartiennent au genre Streptococcus Rosenbach, tribu des Streptococceae Trevisan, famille des Lactobacillaceae Winslow et al., le groupe comprenant deux espèces :

- Streptococcus faecalis Andrewes et Horder et ses variétés - zymogenes Mc Callum et Hastings - liquefaciens Sternberg.

- Streptococcus durans Sherman et Wing.

Toutefois la plupart des études relatives aux entérocoques (Barnes et al., 1956, Deibel, 1964 ; Buttiaux et al., 1969) mentionnent en outre l'espèce Streptococcus faecium Orla-Jensen.

Les entérocoques sont souvent désignés sous l'appellation « Streptocoques du groupe D », mais celle-ci regroupe également deux autres espèces Streptococcus bovis Orla-Jensen et Streptococcus equinus Andrewes et Horder.

Ces bactéries, proches des bactéries lactiques par leur aptitude à l'acidification et à la coagulation du lait (Sherman, 1937), sont présentes en proportions très appréciables dans tous les fromages. Ainsi, leur nombre atteint $10^{7}$ à $10^{8}$ par gramme dans les fromages de Camembert (Lenoir, 1962) et de Roquefort (Devoyod, 1969). Dans les pâtes de Cheddar, elles forment la majeure partie de la flore capable de se développer à basse température, et des nombres de germes atteignant $10^{8}$ par gramme ont été rapportés (Clark et Reinbold, 1967). Dans les fromages les espèces le plus souvent rencontrées paraissent être Str. faecalis et ses variétés (Clark et Reinbold, 1966-1967 ; Devoyod, 1969) et Str. durans (Clark et Reinbold, 1966-1967). Par leur activité biochimique, notamment leur action sur les protéines et leurs produits de dégradation, ces micro-organismes peuvent contribuer utilement à la maturation des fromages et à la formation de l'arôme. 
L'action protéolytique de Str. faecalis var. liquefaciens est notamment bien connue (Bleisweis et Zimmerman, 1964; Shugart et Beck, 1964 ; Casas et Zimmerman, 1969 ; Somkuti et Babel, 1969), et cette action, outre son influence propre, peut avoir des effets indirects car il a été clairement montré que les produits libérés stimulent le développement et l'activité des streptocoques lactiques et des leuconostoc (Devoyod et Muller, 1969 ; Devoyod et Desmazeaud, 1970).

L'idée d'employer les streptocoques du groupe D en fromagerie n'est pas nouvelle. Divers essais, comportant l'addition au levain de Str. faecalis var. liquefaciens, avaient été réalisés avant la guerre avec des résultats plus ou moins encourageants (Lane, 1934 ; Hansen, 1937 ) ; ils ont été repris par la suite (Tittsler et al., 1948 ; Deane, 1951). L'emploi des streptocoques du groupe D a eu pour principal objectif la mise au point de nouveaux procédés de fabrication du Cheddar. Ainsi, une association Str. lactis - Str. faecalis permet d'obtenir un fromage de maturation plus rapide et de meilleure saveur que ceux fabriqués avec les levains classiques (Dahlberg et Kosikowski, 1948 a, 1948 b, 1949 ; Freeman, 1959 ; Zhitkova, 1964). La mise en œuvre de levains de Str. durans est également à la base de procédés accélérés de fabrication (Walter et al., 1953, 1956 ; Czulak et al., 1954 ; Czulak et Hammond, 1956) qui permettent l'obtention de fromages de bonne qualité et de caractéristiques normales (Fryer, 1969). Dans les fabrications de pâtes cuites l'emploi de Str. faecalis comme bactérie du levain a été préconisé soit pour combattre le défaut "mille trous " (Kurmann, 1966, 1968), soit pour améliorer la saveur et l'arôme des fromages obtenus à partir de lait pasteurisé (Storgards et al., 1950, Storgards et Hietaranta, 1950).

A notre connaissance, l'emploi des streptocoques du groupe D comme bactéries du levain n'a pas fait l'objet d'essais systématiques dans les fabrications fromagères françaises. Les résultats obtenus à l'étranger semblent cependant montrer que l'utilisation de souches judicieusement choisies de Str. faecalis, Str. faecalis var. liquefaciens, Str. faecium, Str. durans, peut contribuer à assurer à la fois une acidification satisfaisante du caillé et un développement de saveur et d'arôme convenable.

En vue de procéder à de tels essais une collection de souches d'entérocoques de diverses origines a été constituée et, après identification des souches, leurs principaux caractères technologiques, plus particulièrement leurs aptitudes à l'acidification, à la production de composants d'arôme et à la dégradation des matières azotées, ont été déterminés.

\section{I. - CONSTITUTION D'UNE COLLECTION}

Pour estimer les aptitudes technologiques du groupe il convenait de disposer d'un nombre suffisant de souches prélevées sur des substrats de natures et d'origines différentes. 


\subsection{Protocole expérimental}

\subsubsection{Isolement des souches}

Les isolements ont été effectués à partir de divers substrats : laits crus de mélange, fromages de lait cru d'origines et de types différents (Camembert, Roblochon, Carré de l'Est...), eaux de rivière, eaux fluvio-marines, fécès de plusieurs espèces animales (mouton, porc, cheval, veau).

Après dissociation éventuelle dans le citrate de sodium à 2 p. 100 (fromages) ou dans le carbonate de sodium à $1 \mathrm{p} .100$ (fécès), les échantillons prélevés sont directement ensemencés sur le milieu présomptif de Litsky et al. (1953) modifié par Buttiaux*, de composition suivante : tryptose (Difco), 2 p. 100 ; glucose, 0,5 p. 100 ; chlorure de sodium, 0,5 p. 100 ; phosphate monopotassique, 0,27 p. 100 ; phosphate bipotassique, 0,27 p. 100 ; azothydrate de sodium, 0,02 p. 100 ; pH 6,8. Ce milieu a été choisi de préférence à celui de Hajna et Perry (1943) jugé trop inhibiteur (Buttiaux, 1958 b). Les tubes présentant un louche, après $24 \mathrm{~h}$ d'incubation à $37^{\circ} \mathrm{C}$, sont considérés comme susceptibles de contenir un streptocoque du groupe D. Ils sont alors ensemencés sur le milieu confirmatif de Litsky et al. (1955) modifié par Buttiaux*, qui, par comparaison avec le milieu présomptif, contient de l'éthyl violet $(0,5 \mathrm{ppm})$ et 0,03 p. 100 d'azothydrate de sodium. Sur ce milieu, l'apparition d'un trouble et (ou) la formation au fond du tube d'une pastille violette, après $48 \mathrm{~h}$ d'incubation à $37^{\circ} \mathrm{C}$, indiquent la présence d'un entérocoque.

Les cultures en milieu confirmatif de Litsky sont ensemencées sur le milieu de différenciation de Barnes (1956 a, 1956 b) de composition suivante : peptone Evans, 1 p. 100 ; extrait de viande, 1 p. 100 ; glucose, 1 p. 100 ; chlorhydrate de triphényl tetrazolium (TTC), 0,01 p. 100 ; acétate de thallium, 0,1 p. 100 ; bacto agar, 1,4 p. 100 ; pH 6,0. Sur ce milieu, de petites colonies, roses ou blanches selon que le TTC est réduit ou non en formosan, avec ou sans auréole, apparaissent après $48 \mathrm{~h}$ de culture à $37^{\circ} \mathrm{C}$. L'aspect des colonies, permet d'effectuer une distinction initiale entre les deux groupes, Str. faecalis et ses variétés d'une part, Str. faecium et Str. durans d'autre part ; en revanche Str. bovis cultive mal (Medrek et Barnes, 1962). Trois à cinq colonies développées sur milieu de Barnes et présentant des caractères différents sont prélevées et ensemencées en bouillon lactosé.

Les isolements effectués à partir d'eaux fluvio-marines** ont été réalisés selon une technique différente. Un volume d'eau, variant de 30 à $200 \mathrm{ml}$ suivant la charge microbienne présumée, est filtré à l'aide d'un appareil Millipore sur membrane de type Coli 5 (diamètre des pores : $0,45 \mu)$. La membrane est déposée ensuite sur le milieu

* Cité in Rev. Ferment. et Ind. alim., 21, 45.

** Eaux prélevées dans l'embouchure de l'Orne. 
sélectif de Slanetz et Bartley (1957) contenant tryptose (Difco), 2 p. 100 ; extrait de levure, 0,5 p. 100 ; glucose, 0,2 p. 100 ; phosphate bipotassique, 0,4 p. 100 ; azothydrate de sodium, 0,04 p. 100 ; chlorhydrate de triphényl tetrazolium, 0,01 p. 100 ; bacto-agar, 1 p. 100 ; $\mathrm{pH}: 7,2$. Après $48 \mathrm{~h}$ d'incubation à $37^{\circ} \mathrm{C}, 3$ à 5 colonies sont prélevées sur chaque boîte et portées sur le milieu confirmatif de Litsky. Un isolement est alors effectué sur milieu de Barnes, une colonie est prélevée et ensemencée en bouillon lactosé.

A partir de chacune des cultures en bouillon lactosé un nouvel isolement sur gélose nutritive a permis d'assurer la pureté des souches (tab. 1).

Cent-vingt-neuf souches ont été ainsi isolées et conservées sur gélose inclinée à $+4^{\circ} \mathrm{C}$.

\subsubsection{Identification des souches}

Chaque souche est d'abord ensemencée deux fois, à 24 h d'intervalle, en bouillon lactosé et cultivée à $37^{\circ} \mathrm{C}$.

Sur les cultures bactériennes de $24 \mathrm{~h}$, un examen microscopique avec coloration au bleu de méthylène et une recherche de catalase ont permis de confirmer l'appartenance des souches au groupe des streptocoques. Les milieux d'identification sont ensuite inoculés à partir de ces cultures.

Pour l'identification des espèces, les caractères physiologiques, biochimiques et sérologiques suivants ont été étudiés (Sherman, 19371938 ; Deibel et al., 1963 ; Deibel, 1964) :

- croissance à $10^{\circ} \mathrm{C}, 45^{\circ} \mathrm{C}$ et $50^{\circ} \mathrm{C}$ par ensemencement en bouillon lactosé préalablement porté à la température choisie. Le développement microbien est apprécié par examen de la turbidité et mesure du $\mathrm{pH}$ de la culture, après $24 \mathrm{~h}$ et $48 \mathrm{~h}$ d'incubation à $45^{\circ} \mathrm{C}$ et $50^{\circ} \mathrm{C}$, après une semaine à $10^{\circ} \mathrm{C}$,

- résistance à un chauffage de $30 \mathrm{mn}$ à $60^{\circ} \mathrm{C}$ selon la technique de Abd-el-Malek et Gibson (1948),

- croissance sur lait à 0,1 p. 100 de bleu de méthylène selon la méthode de Sherman (1937),

- réduction du tellurite de potassium à $1 / 2500$ (Skadhauge, 1950) par inoculation du milieu suivant : bacto peptone, 1 p. 100 ; extrait de viande, 1 p. 100 ; chlorure de sodium, 0,5 p. 100 ; extrait de levure, 0,3 p. 100 ; glucose, 0,5 p. 100 ; bacto agar, 2 p. 100 ; pH : 7,1; à $20 \mathrm{ml}$ de milieu, on ajoute avant emploi $1 \mathrm{ml}$ d'une solution stérile de tellurite de potassium à 0,8 p. 100. Le milieu est coulé en boîte de Pétri et la réduction du tellurite est caractérisée par le développement de colonies noires,

- action sur le lait tournesolé ; les changements caractéristiques du milieu - acidification, réduction, coagulation - sont notés après $24 \mathrm{~h}, 48 \mathrm{~h}$ et une semaine d'incubation à $37^{\circ} \mathrm{C}$, 
TABLEAU 1. - Schéma de l'isolement
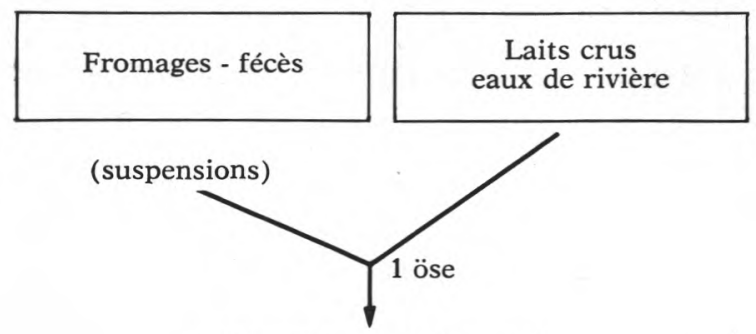

Milieu liquide présomptif

$$
\text { de Litsky }
$$

$\left(24 \mathrm{~h}-37^{\circ} \mathrm{C}\right)$

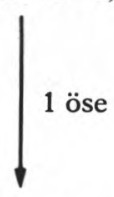

Milieu liquide confirmatif

de Litsky

$\left(48 \mathrm{~h}-37^{\circ} \mathrm{C}\right)$

1 öse

(étalement en stries)

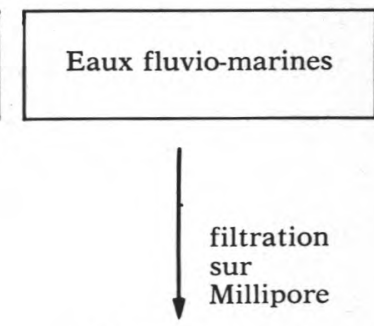

Milieu de Slanetz et Bartley en boîte de Pétri $\left(48 \mathrm{~h}-37^{\circ} \mathrm{C}\right)$

3 à 5 colonies d'aspect différent

Milieu liquide confirmatif de Litsky $\left(48 \mathrm{~h}-37^{\circ} \mathrm{C}\right)$

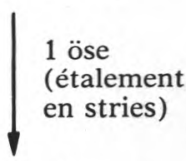

Milieu de Barnes en boîte de Pétri

$\left(48 \mathrm{~h}-37^{\circ} \mathrm{C}\right)$
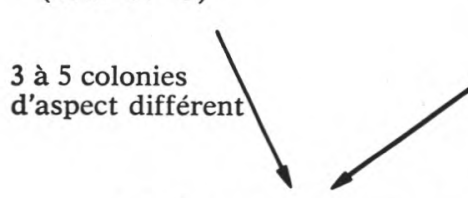

Milieu de Barnes en boîte de Pétri $\left(48 \mathrm{~h}-37^{\circ} \mathrm{C}\right)$

Bouillon lactosé $\left(24 \mathrm{~h}-37^{\circ} \mathrm{C}\right)$

1 öse (étalement en stries)

Gélose nutritive en boîte de Pétri $\left(48 \mathrm{~h}-37^{\circ} \mathrm{C}\right.$ )

$$
\downarrow 1 \text { colonie }
$$

Bouillon lactosé $\left(24 \mathrm{~h}-37^{\circ} \mathrm{C}\right)$

1 öse

Gélose inclinée $\left(24 \mathrm{~h}-37^{\circ} \mathrm{C}\right)$

Conservation à $+4^{\circ} \mathrm{C}$ 
- croissance en bouillon nutritif en présence de 40 p. 100 de bile; l'examen des cultures est fait après $24 \mathrm{~h}$ et $48 \mathrm{~h}$ d'incubation à $37^{\circ} \mathrm{C}$,

- croissance en milieu à 6,5 p. 100 de chlorure de sodium ; le développement microbien est apprécié après une incubation de $48 \mathrm{~h}$ à $37^{\circ} \mathrm{C}$ par examen de la turbidité et mesure du $\mathrm{pH}$,

- aptitude à la fermentation des sucres et polyols : lactose, saccharose, mannitol, sorbitol, arabinose, raffinose, glycérol ; le milieu de base suivant est utilisé : extrait de viande, 0,1 p. 100 ; protéose peptone $\mathrm{n}^{\circ} 3$ (Difco), 1 p. 100 ; chlorure de sodium, 0,5 p. 100 ; rouge de phénol, 0,0018 p. 100 ; bacto agar, 0,1 p. 100 ; pH : 7,6. La solution sucrée est stérilisée par filtration sur bougie Chamberland L 3 et ajoutée au milieu de base à la concentration finale de 0,5 p. 100. L'aptitude fermentaire est notée, après $24 \mathrm{~h}$ et $72 \mathrm{~h}$ d'incubation à $37^{\circ} \mathrm{C}$, en se fondant sur le virage ou le non virage de l'indicateur. Avec le glycérol, le test est réalisé en anaérobiose selon la technique préconisée par Gunsalus et Sherman (1943),

- liquéfaction de la gélatine par ensemencement en piqure sur le milieu de Smith et al. (1952) coulé en boîtes de Pétri ; l'hydrolyse est décelée à l'aide de chlorure mercurique selon la méthode de Frazier (1926) après 4 à $8 \mathrm{j}$ d'incubation à $30^{\circ} \mathrm{C}$,

- hémolyse par ensemencement sur le milieu «Tryptose Blood Agar Base » (Difco no 232) additionné de 5 p. 100 de sang de mouton ; après $24 \mathrm{~h}$ et $72 \mathrm{~h}$ d'incubation à $37^{\circ} \mathrm{C}$, l'apparition ou non d'un halo d'hémolyse est notée,

- étude de la structure antigénique selon la technique de Lancefield (Buttiaux et al., 1969). Lorsque les zones de précipitation étaient peu distinctes, la méthode de Fuller a été utilisée selon le protocole préconisé par l'Institut Pasteur.

\subsection{Résultats}

Les caractères physiologiques et biochimiques des souches soumises à l'identification figurent dans le tableau 2 .

Sur les 129 souches isolées, 46 s'identifient au groupe Str. faecalis, 62 appartiennent à l'espèce Str. faecium, 15 se rattachent à l'espèce Str. durans ; 6 souches sont indéterminées.

Au sein du groupe Str. faecalis, 17 souches hydrolysent la gélatine et peptonisent le lait tournesolé après coagulation. Elles appartiennent à la variété liquefaciens. Parmi les souches restantes, aucune ne produit une auréole franche sur gélose au sang de mouton, et ne peut donc être identifiée à la variété zymogenes; elles se rattachent à l'espèce-type Str. faecalis.

Au total, 32 souches, dont les 17 Str. faecalis var. liquefaciens, présentent tous les caractères de Str. faecalis décrits par Deibel (1964). Les autres diffèrent de l'espèce-type par leur aptitude à la 
TABLEAU 2. - Caractères des streptocoques du groupe $\mathrm{D}$ isolés

\begin{tabular}{|c|c|c|c|c|}
\hline Epreuves & Str. faecalis & $\begin{array}{c}\text { Str. faecalis } \\
\text { var. liquefaciens }\end{array}$ & Str. faecium & Str. durans \\
\hline $\begin{array}{l}\text { Catalase } \\
\text { Aspect des cultures sur gélose au TTC } \\
\text { Culture dans } 40 \mathrm{p} .100 \text { de bile } \\
\text { Culture dans } 6,5 \mathrm{p} .100 \mathrm{de} \mathrm{NaCl} \\
\text { Croissance à } 10^{\circ} \mathrm{C} \\
\text { Croissance à } 45^{\circ} \mathrm{C} \\
\text { Croissance à } 50^{\circ} \mathrm{C} \\
\text { Thermorésistance }\left(60^{\circ} \mathrm{C}-30 \mathrm{~mm}\right) \\
\text { Réduction du lait au bleu de méthy- } \\
\text { lène à } 1 \text { p. } 1000 \\
\text { Culture sur lait tournesolé } \\
\text { Réduction du tellurite de K à } 1 / 2500 \\
\text { Hémolyse } \\
\text { Production d'acide à partir de : } \\
\text { Lactose } \\
\text { Saccharose } \\
\text { Mannitol } \\
\text { Sorbitol } \\
\text { Arabinose } \\
\text { Raffinose } \\
\text { Glycérol } \\
\text { Hydrolyse de la gélatine }\end{array}$ & $\begin{array}{c}- \\
\text { Rouge } \\
+ \\
+ \\
+ \\
+ \\
+ \\
+ \\
+ \\
\text { ARC } \\
+ \\
+\end{array}$ & $\begin{array}{c}\text { Rouge } \\
+ \\
+ \\
+ \\
+ \\
+ \\
+ \\
\text { ARC (Peptonisation) } \\
+ \\
+ \\
+ \\
+ \\
+ \\
+ \\
+ \\
+ \\
+\end{array}$ & 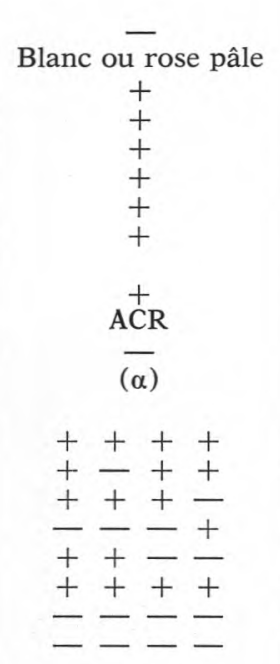 & 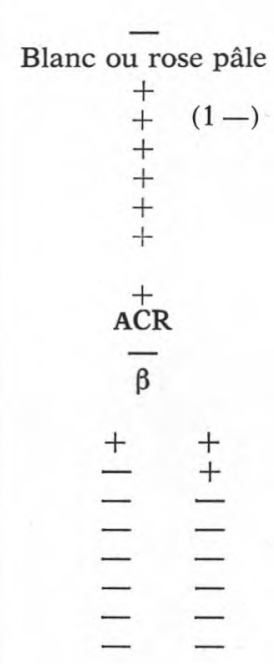 \\
\hline Nombre de souches & $\frac{15}{15 \quad 1 \quad 6 \quad 2 \quad 4}$ & 17 & $\underbrace{35 \quad 19}_{62} 3 \quad 5$ & $\underbrace{11+1}_{15} 3$ \\
\hline
\end{tabular}


fermentation de certains sucres ou polyols. Ainsi, une souche est mannitol (-) et raffinose $(+)$, une est mannitol (-), sorbitol $(-)$ et arabinose $(+), 6$ sont arabinose $(+)$ et raffinose $(+), 2$ arabinose $(+)$ et sorbitol $(-), 4$ souches sont arabinose $(+)$, raffinose $(+)$ et glycérol (-).

Les souches appartenant à l'espèce Str. faecium présentent les caractères spécifiques essentiels : elles ne réduisent pas le tellurite de potassium à $1 / 2500$, elles ne fermentent pas le sorbitol et le glycérol, elles ne réduisent pas le TTC, elles cultivent à $50^{\circ} \mathrm{C}$ et, sur gélose au sang, elles produisent une hémolyse faible de type $\alpha$ avec verdissement du milieu dû à une production d'eau oxygénée. Toutefois, quelques souches se distinguent par leur pouvoir fermentaire ; 19 sont saccharose $(-), 3$ sont arabinose $(-), 5$ sont mannitol (-), arabinose (-) et sorbitol $(+)$.

Les 15 souches identifiées à Str. durans présentent les principaux caractères de l'espèce : elles ne réduisent pas le tellurite de potassium à $1 / 2500$, elles produisent une hémolyse très faible de type $\beta$, elles cultivent à $50^{\circ} \mathrm{C}$, et ne fermentent pas le saccharose, le mannitol, le sorbitol, l'arabinose et le glycérol ; 3 souches cependant sont saccharose $(+)$ et une souche ne cultive pas en présence de 6,5 p. 100 de chlorure de sodium.

\subsection{Discussion}

Le nombre de souches isolées et identifiées à partir des divers substrats, laits crus, fromages, eaux de rivière ou fluvio-marines, fécès, est certes trop limité pour qu'il puisse conférer à la collection un caractère représentatif. Il ne s'agit donc pas d'une étude écologique ; toutefois, la répartition des souches selon leur origine (tab. 3) permet de faire certaines observations.

Dans les produits laitiers, laits crus et fromages, les trois espèces, Str. faecalis, Str. faecium et Str. durans sont présentes en proportions appréciables; le groupe Str. faecalis est notamment bien représenté, et au sein de ce groupe Str. faecalis var. liquefaciens forme une fraction relativement importante. Une telle observation a été faite par Devoyod (1969) sur la flore microbienne du fromage de Roquefort.

Dans les eaux, plus particulièrement celles d'origine fluviomarine, l'espèce Str. faecium apparaît largement dominante ; au contraire, l'espèce Str. durans est absente ou peu représentée. Ces résultats sont en accord avec ceux obtenus par Buttiaux (1958 b) sur les eaux d'alimentation.

En ce qui concerne les fécès, il a été bien établi par divers travaux que la répartition des entérocoques pouvait être très différente d'une espèce animale à une autre. Ainsi, Str. faecalis, fréquemment rencontré dans les fécès d'origine humaine, n'est présent qu'en très faibles proportions chez les ovins et les bovins (Cooper et Ramadan, 1955 ; Willsens et Buttiaux, 1958) et il est pratiquement absent dans 
$T A B L E A U 3$ - Répartition des souches isolées selon leur origine

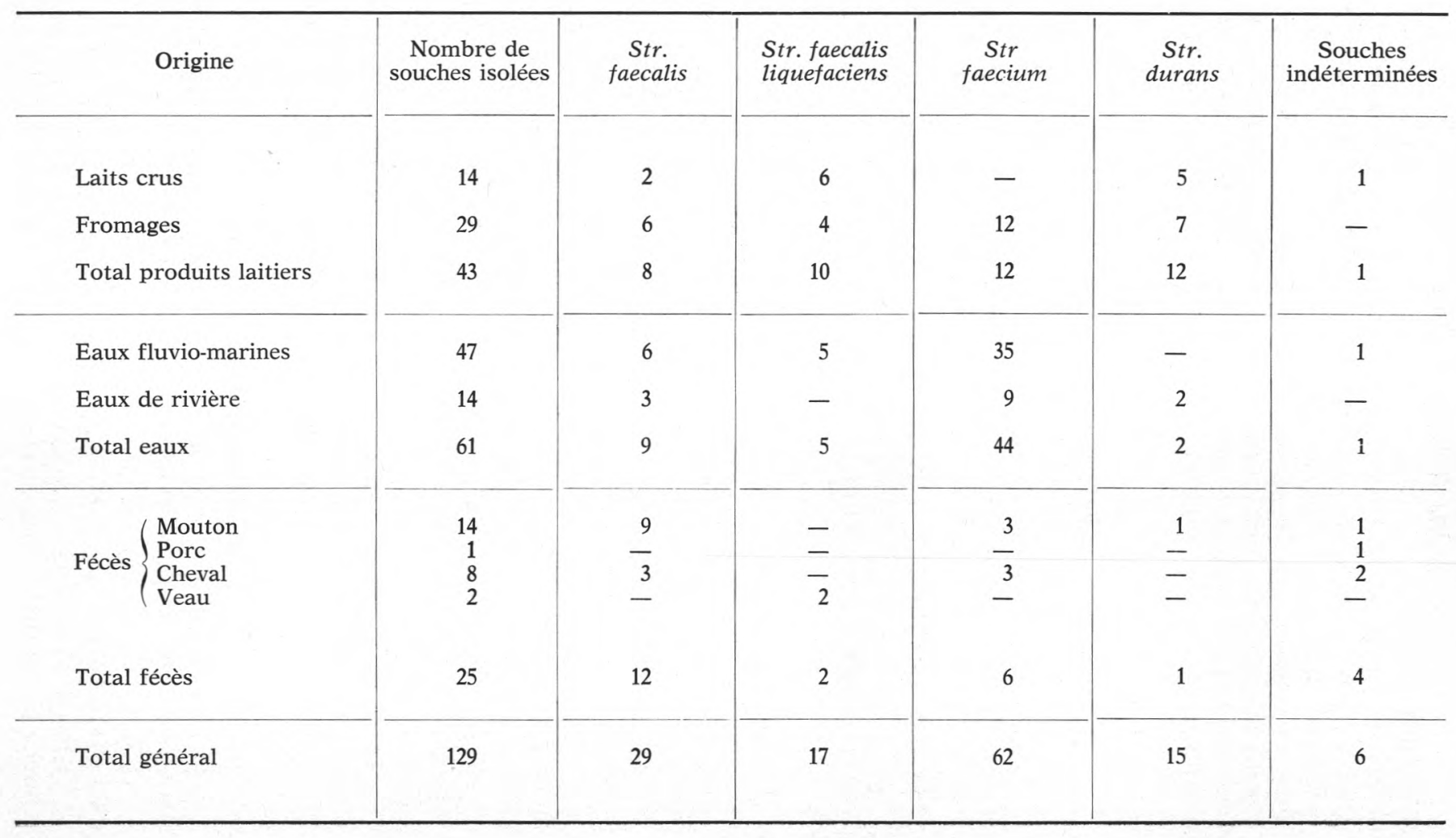


les fécès de porc où Str. faecium est largement dominant (Barnes et al., 1956 ; Raibaud et Caulet, 1957). Le nombre de souches isolées de ces substrats est ici trop restreint pour qu'il soit possible de tirer des conclusions de portée générale. On peut retenir cependant la présence dominante des espèces Str. faecalis et Str. faecium dans les fécès de mouton et de cheval. L'observation concernant les fécès d'ovins ne s'accorde donc pas avec les travaux antérieurs. Cette différence pourrait s'expliquer par un facteur de variation lié au mode d'alimentation et aux conditions de vie des animaux (Buttiaux, 1958 a).

\section{II. - APTITUDE A L'ACIDIFICATION}

L'un des caractères technologiques essentiels des bactéries des levains de fromagerie est leur aptitude à l'acidification du lait qui dépend de l'aptitude à la fermentation du lactose et de la résistance à l'acidité développée.

\subsection{Protocole expérimental}

\subsubsection{Etude portant sur l'ensemble de la collection}

Afin de n'étudier et de ne conserver que les souches présentant une aptitude acidifiante suffisante, l'ensemble de la collection a été éprouvé par une mesure du $\mathrm{pH}$ des cultures sur lait écrémé autoclavé, après $24 \mathrm{~h}$ d'incubation à $37^{\circ} \mathrm{C}$.

Avant d'être testées, les souches sont repiquées deux fois sur lait, à $24 \mathrm{~h}$ d'intervalle, et cultivées à $37^{\circ} \mathrm{C}$.

Elles sont ensuite ensemencées au taux de 1 p. 100 en erlenmeyers contenant $100 \mathrm{ml}$ de lait écrémé reconstitué, stérilisé par autoclavage (120 $\mathrm{C}-12 \mathrm{mn})$.

Après $24 \mathrm{~h}$ d'incubation à $37^{\circ} \mathrm{C}$, le pH est mesuré directement sur les cultures à l'aide d'un pH-mètre Metrohm E 353 muni d'une électrode de verre.

Après cette épreuve, qui a porté sur 123 souches, seules ont été conservées pour une étude plus complète les souches abaissant le $\mathrm{pH}$ des cultures à au moins 5,50 pour Str. faecalis et Str. faecium, 5,60 pour Str. durans.

\subsubsection{Etablissement des courbes d'acidification}

Les courbes d'acidification ont été établies sur les souches répondant aux conditions définies dans l'épreuve précédente.

Les souches sont au préalable repiquées deux fois sur lait, à 24 h d'intervalle.

Elles sont ensuite ensemencées au taux de 1 p. 100 en erlenmeyers contenant $100 \mathrm{ml}$ de lait écrémé autoclavé et cultivées à $37^{\circ} \mathrm{C}$. 
L'acidification des cultures est suivie par mesure du $\mathrm{pH}$ sur un volume de $1 \mathrm{ml}$ prélevé à l'aide d'une pipette stérile aux temps $0,2,5,8,12,16,24,48$ et $72 \mathrm{~h}$. Les mesures de $\mathrm{pH}$ sont effectuées dans des conditions analogues à celles précisées précédemment.

\subsection{Résultats}

L'épreuve rapide de sélection portant sur les 123 souches d'entérocoques identifiées a mis en évidence des différences notables dans l'aptitude à l'acidification.

Ces différences sont illustrées par les histogrammes et les polygones de fréquence des figures $1,2,3,4$ et 5 qui correspondent respectivement à l'ensemble de la collection (fig. 1), et à chacune des espèces, Str. faecalis (fig. 2), Str. faecalis var. liquefaciens (fig. 3), Str. faecium (fig. 4), et Str. durans (fig. 5).

On observe ainsi sur les cultures de $24 \mathrm{~h}$ à $37^{\circ} \mathrm{C}$ des valeurs de $\mathrm{pH}$ qui oscillent entre 4,8 et 6,4 pour l'ensemble des souches, et, pour chacune des espèces, de 4,8 à 6,3 pour Str. faecalis, de 4,8 à 5,9 pour Str. faecalis var. liquefaciens, de 5,0 à 6,4 pour Str. faecium et de 5,2 à 6,2 pour Str. durans. Un très petit nombre de souches est capable d'acidifier le lait autoclavé à un pH inférieur à 5,0.

L'épreuve de sélection a finalement permis de retenir 37 souches d'entérocoques qui répondent aux conditions fixées, à savoir : aptitude à acidifier le lait à $\mathrm{pH}$ inférieur ou égal à 5,5 pour Str. faecalis et Str. faecium, à $\mathrm{pH}$ inférieur ou égal à 5,6 pour Str. durans.

Pour chacune de ces 37 souches la courbe d'acidification sur lait autoclavé a été établie. On trouvera sur la figure 6 les courbes moyennes calculées pour chacune des quatre espèces d'entérocoques.

\subsection{Discussion}

Les histogrammes et les polygones de fréquence des figures 2, 3 , 4 et 5 tendent à montrer que, chez chaque espèce, la répartition des souches en fonction de leur pouvoir acidifiant n'est pas unimodale. En effet, à côté du maximum correspondant aux classes de $\mathrm{pH}$ où figure le plus grand nombre de souches, on observe au moins un " pic secondaire » qui regroupe les souches se situant dans des classes sensiblement différentes. Ceci est particulièrement net pour le groupe Str. faecalis et l'espèce Str. faecium.

Une autre constatation apparaît clairement : les niveaux moyens d'acidification exprimés par les $\mathrm{pH}$ des cultures sur lait de $24 \mathrm{~h}$ sont assez peu différents d'une espèce à l'autre en ce qui concerne Str. faecalis, Str. faecium et Str. durans. En revanche, Str. faecalis var. liquefaciens se distingue nettement. Les niveaux moyens sont en effet proches de 5,7 pour Str. faecalis, 5,2 pour Str. faecalis var. liquefaciens, 5,7 pour Str. faecium et 5,6 pour Str. durans. 


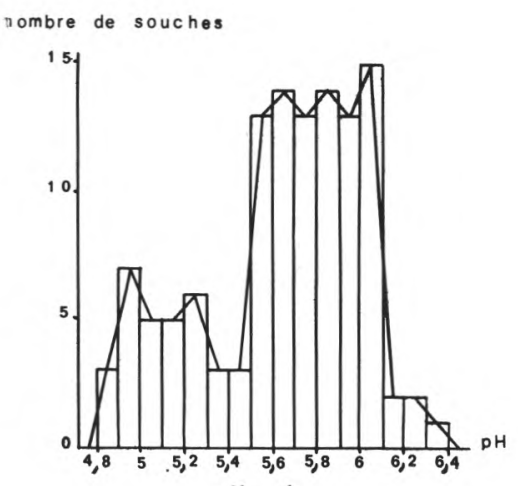

fig. 1

Distribution des souches d'entérocoques

nombre de souches

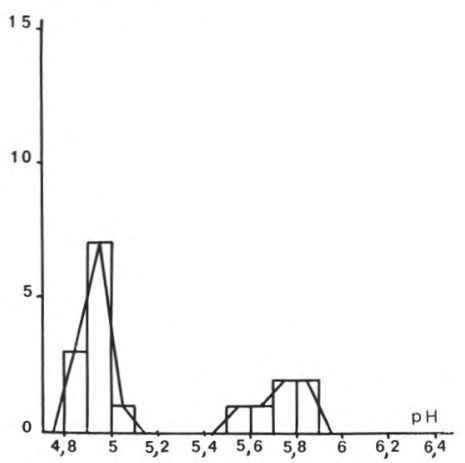

fig. 3

Distribution des souches de Str. faecalis var. liquefaciens nombre de souches

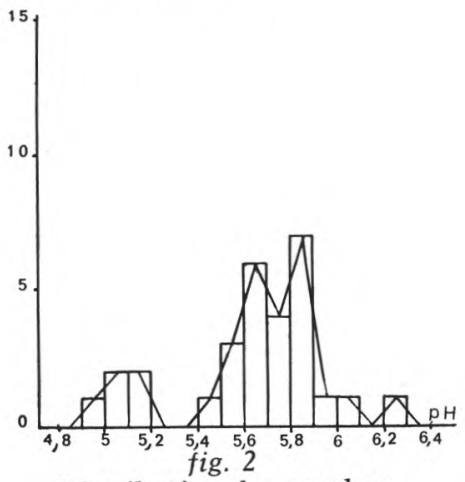

Distribution des souches de Str. faecalis

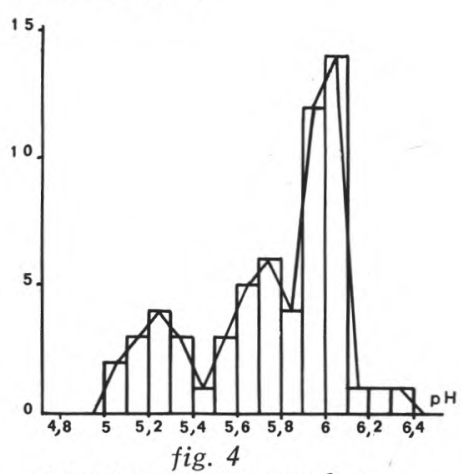

Distribution des souches de Str. faecium

nombre de souches

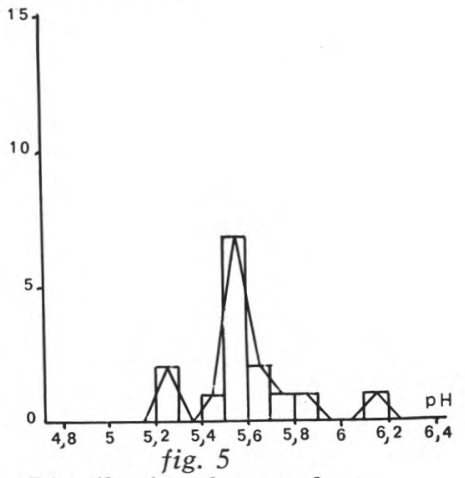

Distribution des souches en fonction de leur pouvoir acidifiant sur milieu lait écrémé autoclavé

Distribution des souches de Str. durans

- en ordonnées : le nombre de souches appartenant aux différentes classes

- en abscisses : les classes d'activité exprimées en unités pH. 
Le choix des pH-limites, 5,5 pour le groupe Str. faecalis et Str. faecium, 5,6 pour Str. durans peut, a priori, sembler arbitraire. L'examen des figures $2,3,4$ et 5 , et notre objectif de travail, à savoir la sélection d'un certain nombre de souches d'entérocoques en vue de leur utilisation dans les levains de fromagerie, l'expliquent aisément.

Parmi les aptitudes technologiques des souches l'un des premiers critères de choix est naturellement l'aptitude à l'acidification. Il nous fallait donc fixer un $\mathrm{pH}$-limite assez bas mais ce $\mathrm{pH}$ devait cependant être compatible avec le pouvoir acidifiant de chaque espèce afin de conserver un nombre suffisant de souches en vue de l'étude d'autres caractères, la production de composants d'arôme et l'activité protéasique notamment. Le pH-limite 5,5 permet de retenir $65 \mathrm{p} .100$ des souches de Str. faecalis var. liquefaciens, environ 20 p. 100 des souches de Str. faecalis et de Str. faecium, mais il élimine la presque totalité des souches de Str. durans; près de la moitié des souches de cette espèce se situe en effet dans la classe de $\mathrm{pH}$ 5,5 - 5,6.

Le tableau 4 rend compte de la répartition des souches sélectionnées sur cette base en fonction de leur origine. Il est intéressant de comparer ces données avec celles précisant la répartition des souches de la collection ( $\operatorname{tab} .3$ ). On observe que les souches isolées à partir de produits laitiers présentent globalement une plus grande aptitude à l'acidification. Ce résultat, non inattendu, n'est pas seulement dû au fait que les espèces les plus acidifiantes sont trouvées en relativement plus grand nombre dans les produits laitiers; au sein même d'une espèce une proportion plus grande de souches acidifiantes a été en effet isolée à partir de laits ou de fromages.

Les courbes moyennes d'acidification établies, pour chaque espèce, avec les 37 souches sélectionnées peuvent être comparées entre elles et avec les courbes d'acidification de quelques souches de Streptococcus lactis de la collection du laboratoire (fig. 6). Ces graphiques mettent clairement en évidence les différences d'aptitude à l'acidification de chacune des espèces d'entérocoques, différences qui apparaissent non pas tant sur le $\mathrm{pH}$ final des cultures que sur la vitesse d'acidification, l'espèce Str. faecalis var. liquefaciens étant de ce point de vue particulièrement active.

On peut aussi observer que les streptocoques lactiques ont un pouvoir acidifiant finalement supérieur à celui des entérocoques ; toutefois, dans les 6 premières heures de culture, les entérocoques sont aptes à provoquer une acidification plus rapide du milieu. Il y a là un facteur favorable pour leur emploi dans les levains de fromagerie.

Il convenait cependant de démontrer que les différences observées entre les profils des courbes moyennes d'acidification des diverses espèces étaient réellement significatives. Par ajustement de la courbe moyenne à un modèle théorique et comparaison des 


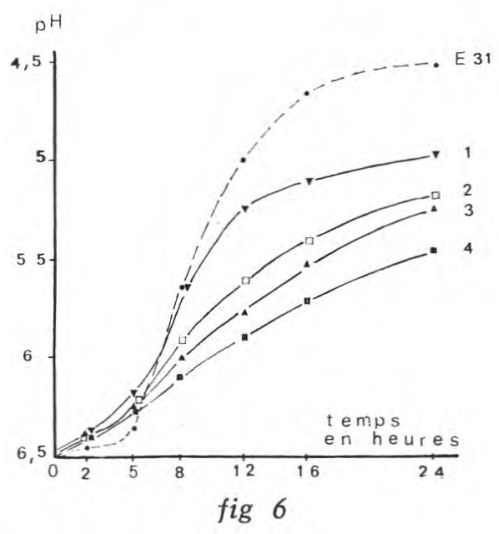

Courbes moyennes d'acidification des différentes espèces d'entérocoques : Comparaison avec les courbes d'acidification des streptocoques lactiques

— en ordonnées : pH des cultures en milieu lait écrémé autoclavé

- en abscisses : temps de culture exprimé en heures.

$$
\begin{gathered}
\text { Entérocoques }\left\{\begin{array}{c}
\boldsymbol{\nabla} 1: \text { Str. faecalis var. liquefaciens } \\
\square 2: \text { Str. faecalis } \\
\mathbf{\Delta} 3: \text { Str. faecium } \\
4: \text { Str. durans }
\end{array}\right. \\
\text { Streptocoques lactiques } \quad \text { - E } 31: \text { Str. lactis }
\end{gathered}
$$

paramètres de ces modèles entre eux, les lois de la statistique établissent que les différences enregistrées entre les temps $5 \mathrm{~h}$ et $24 \mathrm{~h}$, sont bien significatives. Il semble donc y avoir effectivement chez les entérocoques une aptitude à l'acidification caractéristique de l'espèce.

\section{III. - APTITUDE A LA PRODUCTION DE COMPOSANTS D'AROME : DIACETYLE, ACETALDEHYDE, ACETOINE*}

Dans le cadre de cette étude il ne pouvait être envisagé de déterminer l'aptitude des entérocoques à la production des divers composants susceptibles d'intervenir dans l'arôme des fromages. Ne pouvaient non plus être envisagées la séparation et l'estimation des divers aldéhydes et cétones produits. Nous avons retenu le dosage de quelques composés carbonylés témoins, diacétyle, acétaldéhyde,

* Etude réalisée avec la collaboration technique de Michèle Schmidt. 


\section{TABLEAU 4. - Répartition des souches conservées selon leur origine}

\begin{tabular}{|c|c|c|c|c|c|}
\hline Origine & $\begin{array}{c}\text { Nombre de } \\
\text { souches conservées }\end{array}$ & $\begin{array}{l}\text { Str. } \\
\text { faecalis }\end{array}$ & $\begin{array}{l}\text { Str. faecalis } \\
\text { var. liquefaciens }\end{array}$ & $\begin{array}{c}\text { Str. } \\
\text { faecium }\end{array}$ & $\begin{array}{l}\text { Str. } \\
\text { durans }\end{array}$ \\
\hline Laits crus & 8 & 1 & 5 & - & 2 \\
\hline Fromages & 14 & 1 & 3 & 5 & 5 \\
\hline Total produits laitiers & 22 & 2 & 8 & 5 & 7 \\
\hline Eaux fluvio-marines & 12 & 2 & 3 & 7 & - \\
\hline Eaux de rivière & 2 & - & - & 1 & 1 \\
\hline Total eaux & 14 & 2 & 3 & 8 & 1 \\
\hline Fécès $\left\{\begin{array}{l}\text { Mouton } \\
\text { Porc } \\
\text { Cheval } \\
\text { Veau }\end{array}\right.$ & $\frac{1}{-}$ & $\frac{1}{-}$ & $\begin{array}{l}- \\
- \\
-\end{array}$ & $\begin{array}{l}- \\
- \\
-\end{array}$ & $\begin{array}{l}- \\
- \\
-\end{array}$ \\
\hline Total fécès & 1 & 1 & 0 & 0 & 0 \\
\hline Total général & 37 & 5 & 11 & 13 & 8 \\
\hline
\end{tabular}


acétoïne, composés pris comme indices du potentiel d'activité métabolique des souches en milieu lait.

\subsection{Protocole expérimental}

\subsubsection{Choix des souches; préparation des cultures}

Les 37 souches de notre collection de streptocoques du groupe D sélectionnées sur la base de leur aptitude à l'acidification ont été éprouvées.

Avant essai, chaque souche est repiquée deux fois à 24 h d'intervalle sur lait écrémé autoclavé, et incubée à $37^{\circ} \mathrm{C}$. Elle est ensuite inoculée au taux de 1 p. 100 dans $100 \mathrm{ml}$ de lait autoclavé et le milieu est maintenu $16 \mathrm{~h}$ à $37^{\circ} \mathrm{C}$. Sur les cultures, les teneurs en diacétyle, acétaldéhyde et acétoïne sont estimées.

\subsubsection{Dosage du diacétyle}

Le dosage du diacétyle a été effectué selon la méthode de Owades et Jakovac (1963) modifiée par Pack et al. (1964).

Après entraînement par l'azote, le diacétyle est converti en diméthylglyoxine par action de l'hydroxylamine en solution tamponnée. Par réaction avec le sulfate ferreux, la diméthylglyoxine forme un complexe coloré et l'intensité de la coloration est mesurée avec un spectrophotomètre Beckman DU à $530 \mathrm{~m} \mu$. La concentration en diacétyle est obtenue par comparaison avec une courbe étalon établie avec la diméthylglyoxine dans l'intervalle des concentrations de 0 à $50 \mu \mathrm{g}$ par $\mathrm{ml}$ de mélange réactionnel.

\subsubsection{Dosage de l'acétaldéhyde}

Le dosage de l'acétaldéhyde a été réalisé selon la méthode de Lindsay et Day (1965) au 3-méthyl-2-benzothiazolone. En présence d'une hydrazone, les aldéhydes développent en milieu acide une coloration bleue dont l'intensité est mesurée au spectrophotomètre à $666 \mathrm{~m} \mu$. La concentration est calculée en se reportant à une courbe étalon préalablement établie avec de l'acétaldéhyde pur dans l'intervalle des concentrations de 0 à $50 \mu \mathrm{g}$ par $\mathrm{ml}$ de mélange réactionnel.

\subsubsection{Dosage de l'acétoüne}

Le taux d'acétoïne est estimé selon la méthode colorimétrique de Westerfeld (1945) et Brandl (1960) modifiée par Snudden et al. (1965). Cette méthode repose sur le test bien connu de VogesProskauer rendu plus sensible par addition d' $\alpha$-naphtol et de créatine. A l'air, en milieu alcalin, l'acétoïne est oxydée et, en présence des réactifs, développe une coloration rose dont l'absorption est mesurée au spectrophotomètre à $525 \mathrm{~m} \mu$. La réaction concerne l'ensemble diacétyle - acétoïne ; sa concentration est calculée par comparaison avec une courbe étalon établie avec du diacétyle pur dans l'intervalle des concentrations de 0 à $200 \mu \mathrm{g}$ par $\mathrm{ml}$ de mélange réactionnel. 
Dans ces trois déterminations, les blancs utilisés pour régler le zéro du spectrophotomètre sont des laits écrémés autoclavés non ensemencés qui sont traités exactement de la même façon que les cultures à tester.

Toutes les analyses ont été faites en triple, chaque souche étant ensemencée sur trois fioles de lait écrémé autoclavé et incubée $16 \mathrm{~h}$ à $37^{\circ} \mathrm{C}$

\subsection{Résultats}

\subsubsection{Justification du mode opératoire}

Le temps d'incubation adopté, $16 \mathrm{~h}$ à $37^{\circ} \mathrm{C}$, n'a pas seulement été choisi pour des raisons de commodité. Certains auteurs, Keenan et al. (1966) notamment, ont en effet montré, sur des levains lactiques, que les taux de diacétyle et d'acétaldéhyde produits par les micro-organismes tendent à augmenter jusque vers la $12^{\text {me }}-14^{\text {me }} \mathrm{h}$, puis à diminuer dans des proportions variables selon les souches, au cours des $12 \mathrm{~h}$ qui suivent. Afin de nous placer toujours dans les mêmes conditions, le temps de $16 \mathrm{~h}$ a été également utilisé pour éprouver l'aptitude des souches à la production d'acétoïne.

La récupération des composants et la reproductibilité des résultats ont été estimées, pour chacun des trois dosages réalisés, sur cinq déterminations. Les taux moyens suivants ont été obtenus. Ils peuvent être considérés comme relativement satisfaisants pour des analyses en série portant sur des composés qui sont produits - et qui peuvent agir comme facteurs d'arôme - à faibles doses.

\begin{tabular}{lr|c|c|c}
\hline & Diacétyle & Acétaldéhyde & Acétoïne \\
\cline { 3 - 4 } & & & & - \\
Récupération & p. 100 & 83 & 80 & 91 \\
Reproductibilité p. 100 & 86,5 & 86 & \\
\hline
\end{tabular}

Le choix de la méthode d'Owades et Jakovac (1963) pour le dosage du diacétyle et de la méthode de Lindsay et Day (1965) pour celui de l'acétaldéhyde a l'avantage de permettre la détermination simultanée, c'est-à-dire sur une même culture, des niveaux de production des deux composés d'arôme. Les deux méthodes reposent en effet sur l'entraînement par l'azote de ces deux composés volatils qui sont ensuite piégés dans des réactifs spécifiques.

\subsubsection{Production de diacétyle, d'acétaldéhyde et d'acétoïne}

Les teneurs en diacétyle, acétaldéhyde et acétoïne produites dans les cultures sur lait écrémé autoclavé, après $16 \mathrm{~h}$ d'incubation à $37^{\circ} \mathrm{C}$, 
par les différentes souches de streptocoques du groupe D essayées, sont mentionnées dans les tableaux 5 et 6 . Sur ces tableaux les $\mathrm{pH}$ de fin de culture sont également notés.

La présentation groupée des résultats concernant le diacétyle et l'acétaldéhyde rappelle que ces dosages ont été réalisés sur les mêmes cultures.

\subsection{Discussion}

L'aptitude des entérocoques à la production de diacétyle paraît être, dans l'ensemble, assez faible. Il y a des variations importantes d'une souche à l'autre au sein de chacune des espèces, puisque des taux allant de 0 à $0,30 \mathrm{ppm}$ sont observés chez Str. faecalis, de 0 à $0,45 \mathrm{ppm}$ chez Str. faecalis var. liquefaciens, 0 à $0,35 \mathrm{ppm}$ chez Str. faecium, 0,05 à $0,27 \mathrm{ppm}$ chez Str. durans mais il ne semble guère possible d'attribuer à l'une des espèces une aptitude particulière à la production de diacétyle. Il n'apparaît pas non plus y avoir un rapport entre le taux de diacétyle produit et le $\mathrm{pH}$ final des cultures, aussi est-il peu probable que la faiblesse de production du diacétyle soit liée à l'inhibition à $\mathrm{pH}$ faible d'une diacétyle-réductase comme cela a été montré chez les streptocoques lactiques, notamment Str. diacetilactis (Seitz, 1962).

La faible capacité des souches d'entérocoques à la production de diacétyle peut être comparée à celle trouvée, en utilisant la même méthode de dosage, chez les streptocoques lactiques (Bottazzi et Dellaglio, 1967) : 0,1 ppm pour Str. lactis, 0,05 à 2,30 ppm pour Str. cremoris, 0,10 à $2,10 \mathrm{ppm}$ pour Str. thermophilus, 3 à $4 \mathrm{ppm}$ pour Str. diacetilactis, certaines souches de cette dernière espèce pouvant cependant produire jusqu'à $30 \mathrm{ppm}$ de diacétyle.

La capacité de production d'acétaldéhyde présente, elle aussi, quelques variations d'une souche à l'autre mais leur amplitude est limitée, exception faite chez Str. durans. Il semble possible d'attribuer à chaque espèce un niveau moyen de production : $2,3 \mathrm{ppm}$ pour Str . faecalis, 2,6 ppm pour Str. faecalis var. liquefaciens, 1,3 ppm pour Str. faecium et 1,1 ppm pour Str. durans; les coefficients de variation observés pour ces quatre espèces étant respectivement $30,21,22$ et 74 p. 100. Des teneurs en acétaldéhyde comparables ont été signalées sur des cultures de streptocoques lactiques, Str. lactis et Str. cremoris (Harvey, 1960 ; Bottazzi et Dellaglio, 1967) ; en revanche, des niveaux de production légèrement plus élevés ont été trouvés chez Str. thermophilus et les lactobacilles thermophiles (Bottazzi et Vescoco, 1969). Quant à l'espèce Str. diacetilactis, on lui reconnaît une grande aptitude à la production d'acétaldéhyde. Une telle aptitude n'est pas considérée en fromagerie comme un facteur très favorable ; une teneur élevée en acétaldéhyde tend en effet à développer des goûts anormaux, notamment saveur de yoghourt ou saveur «fruitée » (Harvey, 1960 ; Badings et Galesloot, 1962 ; Lindsay et al., 1965). 
TABLEAU 5. - Aptitude des entérocoques à la production de diacétyle et d'acétaldéhyde

\begin{tabular}{|c|c|c|c|c|c|}
\hline Souches & & $\begin{array}{l}\mathrm{pH} \text { de fin } \\
\text { de culture }\end{array}$ & $\begin{array}{l}\text { Diacétyle } \\
(\mathrm{ppm})\end{array}$ & $\begin{array}{l}\text { Acétaldéhyde } \\
(\mathrm{ppm})\end{array}$ & D/A \\
\hline Str. faecalis var. liquefaciens & $\begin{array}{r}\text { L } 17 \\
\text { L } 25 \\
\text { C } 30 \\
\text { L } 38 \\
\text { L } 39 \\
\text { L } 40 \\
\text { F } 43 \\
\text { F } 44 \\
\text { EM } 51 \\
\text { EM } 84 \\
\text { EM } 89 \\
\text { EM } 94\end{array}$ & $\begin{array}{l}4,70 \\
4,80 \\
4,65 \\
4,85 \\
5,15 \\
4,85 \\
4,95 \\
4,80 \\
4,75 \\
4,80 \\
5,10 \\
4,75\end{array}$ & $\begin{array}{l}0,00 \\
0,00 \\
0,05 \\
0,00 \\
0,45 \\
0,05 \\
0,05 \\
0,06 \\
0,00 \\
0,00 \\
0,05 \\
0,04\end{array}$ & $\begin{array}{r}1,05 \\
1,80 \\
>3,50 \\
2,10 \\
3,50 \\
2,65 \\
2,65 \\
2,65 \\
2,98 \\
1,60 \\
3,10 \\
>3,50\end{array}$ & $\begin{array}{l}- \\
\overline{-} \\
\overline{-} \\
0,13 \\
0,01 \\
0,01 \\
0,02 \\
- \\
\overline{-} \\
0,01\end{array}$ \\
\hline Str. faecalis & $\begin{aligned} \text { C } 35 \\
\text { EM } 58 \\
\text { EM } 67 \\
\text { B } 128 \\
\text { L } 133\end{aligned}$ & $\begin{array}{l}5,20 \\
5,40 \\
5,55 \\
5,30 \\
5,00\end{array}$ & $\begin{array}{l}0,00 \\
0,05 \\
0,07 \\
0,20 \\
0,30\end{array}$ & $\begin{array}{l}2,45 \\
2,05 \\
1,55 \\
2,45 \\
2,75\end{array}$ & $\begin{array}{l}\overline{0,02} \\
0,03 \\
0,09 \\
0,11\end{array}$ \\
\hline Str. faecium & $\begin{aligned} & \text { F } 9 \\
& \text { F } 22 \\
& \text { EM } 55 \\
& \text { EM } 60 \\
& \text { EM } 62 \\
& \text { EM } 68 \\
& \text { EM } 75 \\
& \text { EM } 85 \\
& \text { EM } 92 \\
& \text { C } 100 \\
& \text { ER } 113 \\
& \text { C } 142 \\
& \text { C } 143\end{aligned}$ & $\begin{array}{l}5,07 \\
5,30 \\
5,30 \\
5,90 \\
5,35 \\
5,75 \\
5,35 \\
5,25 \\
5,65 \\
5,20 \\
5,25 \\
5,60 \\
5,35\end{array}$ & $\begin{array}{l}0,35 \\
0,11 \\
0,15 \\
0,22 \\
0,00 \\
0,23 \\
0,05 \\
0,09 \\
0,06 \\
0,09 \\
0,12 \\
0,00 \\
0,12\end{array}$ & $\begin{array}{l}1,35 \\
0,80 \\
1,25 \\
1,80 \\
1,40 \\
1,17 \\
1,00 \\
1,06 \\
1,15 \\
1,37 \\
1,52 \\
1,05 \\
1,55\end{array}$ & $\begin{array}{l}0,26 \\
0,13 \\
0,12 \\
0,11 \\
\overline{0,19} \\
0,05 \\
0,08 \\
0,05 \\
0,07 \\
0,10 \\
\overline{0,07}\end{array}$ \\
\hline Str. durans & $\begin{aligned} \text { L } 18 \\
\text { F } 21 \\
\text { C } 32 \\
\text { L } 41 \\
\text { C } 102 \\
\text { ER } 115 \\
\text { F } 122 \\
\text { F } 123\end{aligned}$ & $\begin{array}{l}5,95 \\
5,30 \\
5,80 \\
5,75 \\
5,50 \\
5,75 \\
5,30 \\
5,45\end{array}$ & $\begin{array}{l}0,12 \\
0,16 \\
0,05 \\
0,27 \\
0,06 \\
0,05 \\
0,13 \\
0,10\end{array}$ & $\begin{array}{l}0,75 \\
1,30 \\
0,42 \\
1,35 \\
0,10 \\
0,40 \\
2,75 \\
1,80\end{array}$ & $\begin{array}{l}0,16 \\
0,12 \\
0,10 \\
0,20 \\
0,60 \\
0,12 \\
0,05 \\
0,06\end{array}$ \\
\hline
\end{tabular}


TABLEAU 6. - Aptitude des entérocoques à la production d'acétoïne

\begin{tabular}{|c|c|c|c|}
\hline Souches & & $\begin{array}{c}\mathrm{pH} \text { de } \\
\text { fin de culture }\end{array}$ & $\begin{array}{l}\text { Acétoïne } \\
(\mathrm{ppm})\end{array}$ \\
\hline Str. faecalis var. liquefaciens & $\begin{aligned} \text { L } 17 \\
\text { L } 25 \\
\text { C } 30 \\
\text { L } 38 \\
\text { L } 39 \\
\text { L } 40 \\
\text { F } 43 \\
\text { F } 44 \\
\text { EM } 51 \\
\text { EM } 89 \\
\text { EM } 94\end{aligned}$ & $\begin{array}{r}4,80 \\
5,00 \\
4,80 \\
5,07 \\
5,20 \\
5,00 \\
5,20 \\
5,20 \\
4,95 \\
5,15 \\
4,95\end{array}$ & $\begin{array}{l}18 \\
31 \\
14 \\
30 \\
54 \\
56 \\
35 \\
44 \\
14 \\
14 \\
28\end{array}$ \\
\hline Str. faecalis & $\begin{array}{c}\text { C } 35 \\
\text { EM } 58 \\
\text { EM } 67 \\
\text { B } 128 \\
\text { L } 133\end{array}$ & $\begin{array}{l}5,30 \\
5,35 \\
5,65 \\
5,55 \\
5,30\end{array}$ & $\begin{array}{l}19 \\
13 \\
12 \\
34 \\
43\end{array}$ \\
\hline Str. faecium & $\begin{array}{c}\text { F } 9 \\
\text { F } 22 \\
\text { EM } 60 \\
\text { EM } 62 \\
\text { EM } 68 \\
\text { EM } 85 \\
\text { EM } 92 \\
\text { C } 100 \\
\text { C } 142 \\
\text { C } 143\end{array}$ & $\begin{array}{l}5,25 \\
5,50 \\
5,80 \\
5,40 \\
5,55 \\
5,40 \\
5,70 \\
5,35 \\
5,60 \\
5,40\end{array}$ & $\begin{array}{r}64 \\
7 \\
178 \\
66 \\
165 \\
28 \\
68 \\
67 \\
35 \\
42\end{array}$ \\
\hline Str. durans & $\begin{aligned} \text { L } & 18 \\
\text { F } & 21 \\
\text { C } & 32 \\
\text { L } & 41 \\
\text { C } & 102 \\
\text { EM } & 115 \\
\text { F } & 122 \\
\text { F } & 123\end{aligned}$ & $\begin{array}{l}5,75 \\
5,30 \\
5,80 \\
5,70 \\
5,60 \\
5,70 \\
5,40 \\
5,50\end{array}$ & $\begin{array}{r}76 \\
151 \\
14 \\
69 \\
10 \\
11 \\
98 \\
68\end{array}$ \\
\hline
\end{tabular}


Certains auteurs (Lindsay et Day, 1965) ont relevé l'intérêt du rapport diacétyle/acétaldéhyde (D/A), la valeur de ce rapport déterminant la qualité de-l'arôme développé. En travaillant sur des levains lactiques, ces auteurs admettent que seules les cultures donnant un rapport D/A compris entre 2,2 et 4,4 présentent un arôme favorable. Nos résultats (tab. 5) sont très éloignés de ces valeurs. Remarquons toutefois que sur des souches utilisées comme levains de fromagerie, Bottazzi et Dellaglio (1967) ont trouvé des valeurs analogues : 0,10 pour Str. lactis, 0,8 pour Str. cremoris, 0,3 pour $S t r$. thermophilus, et 0,4 pour Str. diacetilactis ; seules, deux souches de Str. diacetilactis présentaient des rapports atteignant 4,1 et 4,8 .

L'aptitude à la production d'acétoïne (tab. 6) est également très variable d'une souche à l'autre et il ne semble pas y avoir de différences caractéristiques entre les espèces. Str. faecalis et Str. faecalis var. liquefaciens produisent de 12 à $54 \mathrm{ppm}$, Str. faecium de 7 à 178 ppm, Str. durans de 10 à $150 \mathrm{ppm}$. Ces résultats peuvent être comparés à ceux mentionnés par Anantharamaiah et al. (1962) pour deux souches de Str. faecalis var. liquefaciens, cultivées $72 \mathrm{~h}$ à $37^{\circ} \mathrm{C}$; ces auteurs ont noté des productions atteignant 48 et $70 \mathrm{ppm}$. Des quantités analogues (29 à $92 \mathrm{ppm}$ ) ont été également relevées par Bovykina (1969).

Si l'on rapproche ces niveaux de production de ceux observés sur les streptocoques lactiques et les lactobacilles (Lindsay et Day, 1965 ; Lindsay et al., 1965 ; Kennan et al., 1966 ; Habaj et al., 1966) il apparaît que les streptocoques du groupe $D$ possèdent une aptitude sensiblement plus marquée à l'élaboration de l'acétoïne. 